$$
\text { Pontifícia Universidade } \text { Católica }_{\text {Do Rio de Janeiro }}
$$

Yolanda Del Rocío Angulo Paredes

\begin{abstract}
Desenvolvimento e caracterização de dispositivos fosforescentes baseados em compostos orgânicos de baixo peso molecular
\end{abstract}

Tese de Doutorado

Tese apresentada como requisito parcial para obtenção do título de Doutor pelo Programa de Pós-Graduação em Física da PUC-Rio.

Orientador: Prof. Marco Cremona

Rio de Janeiro

Março de 2012 


\section{Desenvolvimento e caracterização de dispositivos fosforescentes baseados em compostos orgânicos de baixo peso molecular}

Tese apresentada como requisito parcial para obtenção do grau de Doutor pelo Programa de Pós-Graduação em Física do Departamento de Física do Centro Técnico Científico da PUCRio. Aprovada pela Comissão Examinadora abaixo assinada.

Prof. Marco Cremona

Orientador

Departamento de Física - PUC-Rio

Profa. Sônia Reanux Wanderley Louro

Departamento de Física - PUC-Rio

Prof. Hermi Felinto de Brito USP - Instituto de Química

Prof. Mamfredo Harri Tabacniks

USP

Profa. Caroline Arantes Silva

INMETRO

Prof. José Eugenio Leal Coordenador(a) Setorial do Centro

Técnico Científico - PUC-Rio 
Todos os direitos reservados. É proibida a reprodução total ou parcial do trabalho sem autorização da universidade, da autora e do orientador.

Yolanda Del Rocío Angulo Paredes

Graduou-se em Física pela Escuela Politécnica Nacional (EPN), em 2007 Quito-Ecuador. Tem experiência na área de Física, com ênfase nas áreas de Física da Matéria Condensada e Espectroscopia óptica e optoeletrônica, atuando principalmente nos seguintes temas: física quântica, caracterização de materiais, superfícies e filmes finos, e fabricação de dispositivos orgânicos.

Ficha Catalográfica

Angulo Paredes, Yolanda Del Rocío

Desenvolvimento e caracterização de dispositivos fosforescentes baseados em compostos orgânicos de baixo peso molecular / Yolanda Del Rocío Angulo Paredes; orientador: Marco Cremona. - 2012.

$211 \mathrm{f.} ; 30 \mathrm{~cm}$

Tese (doutorado)-Pontifícia Universidade Católica do Rio de Janeiro, Departamento de Física, 2012.

Incluí referências bibliográficas.

1. Física - Teses. 2. PHOLED's. 3. Eletrônica orgânica. 4. Filmes finos. 5. Eletroluminescência. 6. Nanotecnologia. 7. Caracterização dos materiais. I. Cremona, Marco. II. Pontifícia Universidade Católica do Rio de Janeiro. Departamento de Física. IV. Título. 
A meus padres amados e meus queridos irmãos e amigos.

"Lo esencial es invisible a los ojos" 


\section{Agradecimentos}

Agradeço a Deus.

Aos meus pais e irmãos pelo carinho e pelo incentivo durante todos esses anos.

Ao Prof. Marco Cremona, pela orientação, paciência e por ter me ajudado a crescer não só a nível Professional, se não, também no pessoal.

Ao Prof. Mark E. Thompson (Departamento de Química, USC), Prof. Hermi F. Brito (Instituto de Química, USP), Prof. Sergio Lima (Departamento de FísicoQuímica, USP), Dra. Wei-Wei, pelas sínteses dos complexos.

Ao Prof. Manfredo H. Tabacniks e Dr. Marcel Dupret (LAMFI-IFUSP) pela amizade, ajuda e colaboração nas análises do RBS.

Aos doutores e bolsistas do Instituto de Metrologia, Qualidade e Tecnologia (INMETRO) em especial ao Prof. Carlos A. Achete, Dra. Erika G. Gravina, Dra. Andrea P. Campos, Dr. Fábio Cacais, Dr. Victor Loayza, Dra. Carol Arantes e Marcos Costa E. Silva e aos antigos integrantes do INMETRO, Dr.Rogerio Machado (DFI/UFS), Dr. Welber G Quirino (UFJF), Dr. Cristiano Legnani (UFJF), que me ensinaram, ajudado e brindado sua amizade ao longo deste trabalho.

Aos professores e técnicos do Departamento de Física e do Departamento de Quimica pelos conhecimentos adquiridos e pelo apoio técnico, em especial a Dra. Sônia R. Louro, Dr. Hélio Carvalho, Dra. Eliane Monsores, Dra. Cassia R. Ponciano, Sra. Márcia Arjona, Sra. Giza, Sr. Welington, Sr. Zaneli e Sra. Eliane Garcia. 
A todos meus amigos queridos que me apoiaram constantemente com conselhos e sua amizade incondicional, em especial aqueles que de alguma forma contribuíram diretamente para a realização desta tese, em especial Emy Niyama, Juan H. Serna, Harold Camargo, Gustavo Lanza, Narely Nicolau, Luiz Felipe Nogueira, Beatriz Saure, Gabriel Lemos e Geronimo Perez.

A Pontifícia Universidade Católica do Rio de Janeiro (PUC-Rio) por proporcionar as condições necessárias para o bom andamento do trabalho.

Ao Centro Latino-Americano de Física (CLAF), ao Conselho Nacional de Desenvolvimento Científico e Tecnológico (CNPq), à Rede de Nanotecnologia Molecular e de Interfaces (Renami), pelo suporte financeiro.

A todos os integrantes do grupo da LOEM. 


\section{Resumo}

Angulo Paredes, Yolanda Del Rocio; Cremona, Marco. Desenvolvimento e caracterização de dispositivos fosforescentes baseados em compostos orgânicos de baixo peso molecular. Rio de Janeiro, 2012. 211p. Tese de Doutorado - Departamento de Física, Pontifícia Universidade Católica do Rio de Janeiro.

Neste trabalho é apresentado o estudo dos dispositivos orgânicos emissores de luz fosforescentes (PHOLED) baseados em compostos de baixo peso molecular. O estudo foi fundamentado nas análises dos complexos baseados no íon de $\operatorname{lr}^{3+}$ : [Ir(t-pzp) $)_{2}$ pic] [fac-Ir(t-bupzp $)_{3}$ ] e outros complexos já estudados anteriormente a base de $\mathrm{Eu}^{3+}$ : [Eu(DBM $\left.)_{3} \mathrm{PHEN}\right] \mathrm{e}$ $\left[\mathrm{Eu}(\mathrm{DBM})_{3} \mathrm{DMSO}_{2}\right]$. No desenvolvimento dos PHOLEDs, a dopagem de uma matriz orgânica com estes complexos reveste uma importância fundamental. Por tanto, como o intuito de estudar e analisar estes sistemas os complexos foram dopados com diversas concentrações em diferentes matrizes orgânicas. Os resultados obtidos revelaram que no caso dos complexos de $\operatorname{~Ir~}^{3+}$ os melhores valores da concentração do complexo encontram-se na faixa 13-15\% em peso. Já no caso dos complexos de íons de terra rara $\left(\mathrm{Eu}^{3+}\right)$ as melhores concentrações devem ser superiores a $15 \%$ em massa, para que ocorra uma transferência de energia eficiente e mantendo o espectro de emissão dos complexos resultando em um aumento da eficiência externa dos dispositivos. Neste trabalho foi desenvolvida também, uma metodologia que, combinando as técnicas de XRF e RBS, permite elaborar um gráfico de calibração para a determinação da concentração efetiva do dopante nos diferentes filmes codepositados. Finalmente, para aumentar a eficiência dos PHOLEDs foi investigado o efeito da vibração do substrato durante a deposição térmica dos filmes. Os resultados indicam uma redução na densidade de aglomerados e na rugosidade, além de uma melhoria na homogeneidade dos filmes depositados. O aumento de $40 \%$ na eficiência do dispositivo fabricados indica que a técnica de vibração é promissora para o desenvolvimento de dispositivos eficientes.

\section{Palavras-chave}

PHOLEDs; Eletrônica orgânica; Filmes finos; Eletroluminescência; Nanotecnologia; Caracterização dos materiais. 


\section{Abstract}

Angulo Paredes, Yolanda Del Rocío; Cremona, Marco (Advisor). Development and characterization of phosphorescent devices based on low molecular weight organic compounds. Rio de Janeiro, 2012. 211p. Doctoral Thesis - Departamento de Física, Pontifícia Universidade Católica do Rio de Janeiro.

This thesis reports the investigation of the phosphorescent organic light emitting devices (PHOLED) based on low molecular compounds. The study was carried out by analyzes the optical and electrical characteristics of thin films of $\mathrm{Ir}^{3+}$ : [Ir(t-pzp) $)_{2}$ pic] and [fac-Ir(t-bupzp $\left.)_{3}\right]$ and other complexes based on $\mathrm{Eu}^{3+}:\left[\mathrm{Eu}(\mathrm{DBM})_{3} \mathrm{PHEN}\right]$ and $\left[\mathrm{Eu}(\mathrm{DBM})_{3}(\mathrm{DMSO})_{2}\right]$. In developing of PHOLEDs, technology, the doping of an organic matrix with these complexes has a fundamental importance. Therefore, in order to investigate these systems ${ }^{3 r^{3+}}$ and $\mathrm{Eu}^{3+}$ organic complexes were used as doping with a variety of concentrations in different organic matrix. The results revealed that in the case of Iridium complexes the best values are in the range 13-15\%. On the other hand, for the rare earth complexes the best concentrations should be greater than $15 \%$ in order to obtain a efficient energy transfer preserving the emission spectrum of the complexes. Furthermore, the use of the doping results in an increased of the external efficiency of the device. In this work, a methodology combining the XRF and RBS techniques was also developed which allows the direct determination of the effective dopant concentration in co-deposited films through a calibration curve. Finally, to increase the efficiency of the PHOLEDs, the effect of mechanical vibration of the substrate on the thin films characteristics during the thermal deposition was investigated. The results indicate a reduction in the clusters density and film roughness, and an improved homogeneity of the deposited films. The $40 \%$ increase in efficiency of the devices fabricated under these conditions is a clear indication that the technology holds promise for the development of efficient devices.

\section{Keywords}

PHOLEDs; Organic Electronics; Thin Films; Electroluminescence; Nanotechnology; Material characterization. 


\section{Sumário}

$\begin{array}{ll}\text { Apresentação } & 26\end{array}$

$\begin{array}{ll}\text { Objetivos gerais } & 27\end{array}$

Objetivos específicos $\quad 27$

1. Introdução 29

1.1. Os OLEDs 31

1.2. Mecanismos de funcionamento do OLED 33

1.2.1. Injeção de carga 33

1.2.2. Transporte de carga $\quad 35$

1.2.3. Recombinação e emissão $\quad 37$

1.3. Matrizes e dopantes 38

2. Teoria 40

2.1. Complexos Emissores $\quad 41$

2.1.1. Transições ópticas dos complexos 41

2.1.2. Regras de conservação do spin 42

2.1.2.1. Acoplamento Spin-Orbita 43

2.1.3. Fosforescência $\quad 47$

2.1.3.1. Complexos baseados em íons de metais de transição do bloco d 47

2.1.3.2. Complexos baseados em íons de terra rara (bloco f) 48

2.1.4. Processos de transferência de energia e carga 50

2.1.4.1. Transferência de energia entre matriz e complexo 51

2.1.4.2. Transferência de carga $\quad 54$

2.2. Filmes finos de semicondutor orgânico $\quad 57$

2.3. Eficiência dos OLEDs 59

3. Procedimentos Experimentais 61

3.1. Materiais utilizados 61

3.2. Preparação dos substratos 65

3.2.1. Substratos de ITO/vidro para a fabricação dos OLEDs 66

3.3. Deposição de filmes finos $\quad 67$

3.3.1. Técnica de evaporação térmica 67

3.3.2. Descrição do sistema de evaporação térmica 68

3.3.3. Implantação da técnica de co-deposição térmica 71

3.3.4. Técnica de vibração do substrato 76

4. Técnicas de análises $\quad 80$ 
4.1. Análises dos filmes crescidos por co-deposição térmica 80

4.2. Análises dos filmes crescidos com a técnica de vibração do substrato 80

4.3. Medição da massa dos filmes depositados 80

4.4. Análises espectroscópicas e ópticas 82

4.4.1. Espectroscopia de retro-espalhamento Rutherford (RBS) 82

4.4.2. Fluorescência de raios-X 84

4.4.3. Espectroscopia de absorção no Infravermelho com Transformada de 86 Fourier

4.4.4. Espectroscopia óptica de absorção 88

4.4.5. Espectroscopia de fluorescência 88

4.4.6. Medidas de eletroluminescência 90

4.5. Análises topográficas e morfológicas 91

4.5.1. Microscopia de Força Atômica (AFM) 91

4.5.2. Microscopia eletrônica de transmissão 92

4.6. Análises eletroquímicas e elétricas 95

4.6.1. Voltametria cíclica 95

4.6.2. Medidas elétricas $\quad 97$

4.7. Cálculo da eficiência em dispositivos emissores de luz 97

$\begin{array}{ll}\text { 5. Resultados } & 101\end{array}$

5.1. Caracterização dos complexos fosforescentes 101

5.1.1. Análises do complexo [Ir(t-pzp) ${ }_{2}$ pic] 104

5.1.2. Caracterização óptica das matrizes 109

5.2. Caracterização da técnica de co-deposição térmica 112

5.2.1. Medida da concentração do $\left[\operatorname{Ir}(\mathrm{ppy})_{3}\right]$ com a técnica RBS 112

5.2.2. Medida da concentração do $\left[\operatorname{Ir}(\mathrm{ppy})_{3}\right]$ com a técnica XRF 115

5.3. Caracterização óptica do sistema matriz:complexo 122

5.3.1. Fotoluminescência 122

$\begin{array}{ll}\text { 5.3.2. PHOLEDS } & 134\end{array}$

5.4. Aumento do desempenho dos OLEDs 160

5.4.1. Caracterização dos filmes orgânicos depositados com a técnica de vibração do substrato 162

5.4.2. Análises TEM dos filmes co-depositados [Ir(ppy)3]:Spiro2-CBP 166

5.4.3. Análises elétricas dos OLEDs fabricados utilizando a técnica de vibração 175

5.4.4. Influência da injeção de carga no desempenho dos OLEDs 179

5.5. PHOLEDs com emissão branca baseados nos complexos de $\mathrm{Ir}^{3+} \quad 183$ 
6. Conclusões 188

6.1. Conclusões 188

$\begin{array}{ll}\text { 6.2. Perspectivas } & 191\end{array}$

7. Produção 193

7.1. Trabalhos publicados durante a tese 193

$\begin{array}{ll}\text { 7.2. Trabalhos submetidos } & 193\end{array}$

7.3. Trabalhos apresentados em congressos nacionais e internacionais 193

8. Referências Bibliográficas 196

$\begin{array}{ll}\text { Apêndice A } & 209\end{array}$

A.1. Estudos do complexo $\left[\operatorname{Ir}(\mathrm{ppy})_{3}\right] \quad 209$ 


\section{Lista de figuras}

Figura 1.1: Esquema de bandas de energia de um material condutor, isolante e semicondutor

Figura 1.2: Exemplos de polímeros conjugados a) polivinilcarbazol, b) polímero tipo PPV - polifenileno vinileno.

Figura 1.3: Estrutura básica do dispositivo orgânico eletroluminescente (OLED)

Figura 1.4: Representação da injeção por tunelamento tipo Fowler-Nordheim e injeção termiônica, em uma estrutura metal/semicondutor-intrínseco/metal, onde $\varphi$ corresponde a altura da barreira de potencial na interface metal/semicondutor e $\mathrm{V}$ ao potencial elétrico aplicado entre os eletrodos.

Figura 1.5: Representação do comportamento J-V para um OLED, e os respectivos tipos de transporte em cada região da curva [25], sendo (1) ôhmico, (2) corrente limitada por carga espacial (SCLC), (3) corrente limitada por cargas aprisionadas (TCL) e (4) corrente limitada por carga espacial livre de armadilhas (TFSCLC).

Figura 1.6: Representação da recombinação de cargas que resultam em éxcitons de caráter singleto e/ou tripleto dependendo da molécula emissora [16].

Figura 2.1: Esquema das bandas de energia e dos processos de absorção e emissão (princípio de Franck-Condon) (gráfico modificado ${ }^{[55]}$ ).

Figura 2.2: (a) Diagrama para os orbitais moleculares para um composto com um único orbital $\pi$, $d$ e $\pi^{*}$ e as respectivas transições $M L C T$ e LC. (b)

Diagrama que apresenta os oito estados de energia que mistura as transições MLCT e $L C^{[16]}$.

Figura 2.3: Diagrama de energia com mecanismos de transferência de energia entre um ligante orgânico e um íon terra-rara ${ }^{[64]}$.

Figura 2.4: Representação dos decaimentos radiativos e não radiativos dos estados de tripleto do sistema doador/receptor. As taxas de transferência direta e inversa $\mathrm{k}_{\mathrm{F}}$ e $\mathrm{k}_{\mathrm{R}}$, respectivamente, são determinadas pela variação da energia livre de Gibbs e da sobreposição molecular. Também são mostradas as taxas de decaimento radiativo do doador e receptor dos estados de tripleto, $\mathrm{k}_{\mathrm{d}}$ e $\mathrm{k}_{\mathrm{a}}$, respectivamente ${ }^{[50]}$. 
Figura 2.5: Esquema que representa como uma carga salta com a presença de um campo elétrico. O campo $\mathrm{F}$ muda o potencial relativo das moléculas por aqF, onde "a" é o espaçamento molecular. Cada molécula é representada como um oscilador harmônico simples e, como descrito no texto, a transferência de carga ocorre na intercepção entre as curvas da distorção molecular. Adaptado de Closs e Miller (1988) ${ }^{[15]}$.

Figura 3.1: Estruturas químicas dos complexos usados como dopantes na camada emissora dos PHOLEDs.

Figura 3.2: Estruturas químicas das moléculas orgânicas utilizadas como matrizes: a) Spiro2-CBP, b) TcTa e c) $\alpha-N P D$.

Figura 3.3: Estruturas químicas dos transportadores de carga:

a) $\alpha$-NPB, b) $\beta-N P B$, c) MTCD, e d) Alq

Figura 3.4: Estrutura química de: a) CuPc e b) BCP

Figura 3.5: Esquema dos substratos com ITO. (a) padrão da geometria do ITO no substrato de vidro, (b) área de emissão de $6 \mathrm{~mm}^{2}$ dos substratos de ITO.

Figura 3.6: Foto do equipamento de deposição térmica (UNIVEX 300) antes da implementação da técnica de co-deposição.

Figura 3.7: Foto da parte interna da evaporadora térmica (UNIVEX 300) com os diversos formatos de cadinhos usados.

Figura 3.8: Diagramas dos porta substratos para a deposição dos filmes finos para a fabricação dos OLEDs. (a) antigo sistema de porta substratos, (b) novo sistema de porta substratos ${ }^{[124]}$ formado por porta substrato (1), porta máscaras (2), máscaras para orgânicos (3) e metais (4).

Figura 3.9: Desenho esquemático da câmara de deposição, indicando as distâncias e as posições dos 2 sensores, do substrato e dos cadinhos.

Figura 3.10: Fotos da parte interna da UNIVEX 300. No lado direito a foto dos eletrodos com os cadinhos e no lado esquerdo toda a câmara interna da evaporadora térmica e junto a ele o controlador de espessura INFICOM.

Figura 3.11: Esquema da técnica de vibração do substrato implementada na câmara de um CVD por K.P. Monhanchandrat e J Uchilt e colaboradores $^{[132]}$.

Figura 3.12: Figura esquemática da câmara de deposição, indicando as posições dos sensores, do alto-falante, do substrato e dos cadinhos dentro da câmara. 
Figura 3.13: Esquema do método de medição da amplitude do alto-falante ${ }^{[133]}$.

Figura 4.1: Espalhamento elástico em ângulo traseiro de um íon de massa $\mathrm{M}_{1}$ por um átomo de massa $\mathrm{M}_{2}$, com $\mathrm{M}_{2}>\mathrm{M}_{1}{ }^{[134,136]}$.

Figura 4.2: Esquema dos componentes internos de um espectrômetro de fluorescência de raios-X, A4Pioneer ${ }^{[140]}$.

Figura 4.3: Espectros de FTIR de filmes finos de duas moléculas orgânicas, respectivamente, matriz e dopante, co-depositados com diferentes concentrações de dopante (complexo baseado em íon de $\mathrm{Ir}^{3+}$ ). $\mathrm{Na}$ figura inserida na parte superior direita do espectro é apresentada a ampliação da região do pico característico do dopante observado em amostras com diferentes concentrações de dopante.

Figura 4.4: Diagrama esquemático do espectrofluorímetro PTI modelo 3027. A região em destaque ilustra como é feita a conexão para a medida de eletroluminescência, (esquema modificado do original ${ }^{[143]}$ )

Figura 4.5: Esquema simplificado do princípio de funcionamento do microscópio eletrônico de transmissão: (a) em modo TEM (feixe paralelo, à esquerda) e (b) STEM (feixe focalizado varrendo a amostra ponto a ponto, à direita). Notar a posição da abertura da objetiva gerando o contraste de difração (feixe paralelo), e a posição dos detectores para a obtenção das imagens nos modos TEM (BF = campo claro) e STEM (BF e HAADF = campo escuro anular por grande ângulo) ${ }^{[147]}$.

Figura 4.6: Diagrama dos tipos de sinais obtidos em modo STEM, (esquema modificado do original ${ }^{[147]}$ )

Figura 4.7: (a) Diagrama de bandas para uma estrutura metal/ orgânico/metal, (b) voltamograma obtido para a determinação do potencial de ionização com referência ao elétrodo padrão de Hidrogênio.

Em destaque, o ponto onde se determina o IP.

Figura 4.8: Esquema utilizado para obter as curvas características I-V de OLEDs ${ }^{[1]}$.

Figura 4.9: Resposta padronizada dos três fotorreceptores sensíveis do olho. Coordenadas de cor pode ser calculado pela sobreposição de cada uma dessas respostas com o espectro de emissão do OLED [151].

Figura 4.10: Gráfico do CIE utilizado para quantificar a sensação do cor. 
Figura 5.1: Espectros de excitação e de emissão do ligante t-pzp a 77K. O espectro de emissão em vermelho (aumentado de um fator 10) na região de emissão dos estados tripleto. A figura também apresenta a estrutura química do ligante $t$-pzp.

Figura 5.2: Formulas estruturais dos isômeros obtidos na síntese do complexo [Ir(t-pzp) ${ }_{2}$ pic]. (a) Trans-pzp (molécula não polar), (b) e (c) Cis-pzp (moléculas polares). Na figura também são mostradas as fotos das soluções dos complexos em diclorometano.

Figura 5.3: Espectros de absorção e emissão a temperatura ambiente de soluções, pó e filme fino do complexo de [Ir(t-pzp) ${ }_{2}$ pic] registrados. Os estados designados como: ${ }^{1,3} L C$ e ${ }^{1,3} M L C T$ correspondem às transições de estado de singleto e tripleto do complexo ${ }^{[187]}$. A linha tracejada em (b) é o espectro de absorção da solução aumentado em fator 100 .

Figura 5.4: Espectros $F T I R$ de filmes de $\left[\operatorname{Ir}(t-p z p)_{2} p i c\right]$, e $\left[\operatorname{Ir}(p p z)_{3}\right]$ com $50 \mathrm{~nm}$ de espessura depositados termicamente em substratos de $\mathrm{KBr}$.

Figura 5.5: Voltamograma obtido para a determinação do potencial de ionização dos complexos com respeito ao eletrodo padrão de Hidrogênio (SHE): a) [Ir(ppy) $\left.)_{3}\right]$ e b) [Ir(t-pzp) ${ }_{2}$ pic]. Em destaque, o ponto onde se determina o IP.

Figura 5.6: Espectros de absorção e emissão a temperatura ambiente e baixa temperatura (8 e 77K) de: (a) Spiro2-CBP, (b) TcTa, (c) $\alpha$-NPD. Os espectros de $8 \mathrm{~K}$ foram obtidos da literatura ${ }^{[16]}$.

Figura 5.7: Espectros RBS dos filmes de $\left[\operatorname{Ir}(\mathrm{ppy})_{3}\right]$ :CuPc co-depositados com diferentes concentrações de $\left[\operatorname{Ir}(\mathrm{ppy})_{3}\right]$. Na parte superior direita, encontra-se a ampliação da região que apresenta as bandas de Irídio e Cobre.

Figura 5.8: Concentração em massa do $\left[\operatorname{lr}(\mathrm{ppy})_{3}\right]$ medido com $R B S\left(f_{\text {RBS }}\right)$ em função da fração relativa do [Ir(ppy $\left.)_{3}\right]$ nominal $(f)$ para filmes co-depositados de $\left[\operatorname{Ir}(\mathrm{ppy})_{3}\right]: \mathrm{CuPc}$.

Figura 5.9: Medidas de espessura e massa dos filmes de $\mathrm{Alq}_{3} \mathrm{com}$ o método de $Q C M$ em função da espessura do filme. Dentro desta curva a foto de um dos filmes de $\mathrm{Alq}_{3}$ excitada com lâmpada UV.

Figura 5.10: Espectro de $X R F$ dos filmes de $\left[\operatorname{lr}(\mathrm{ppy})_{3}\right]$ com três diferentes massas do filme. 
Figura 5.11: Curva de calibração massa do filme vs. intensidade do $X R F$ do sinal Ir.

Figura 5.12: Concentração em massa do $\left[\operatorname{Ir}(\mathrm{ppy})_{3}\right]\left(f_{\mathrm{XRF}}\right)$ para filmes co-depositados de (a) $\left[\operatorname{Ir}(\mathrm{ppy})_{3}\right]$ :Spiro2-CBP e (b) $\left[\operatorname{Ir}(\mathrm{ppy})_{3}\right]$ :CuPc. Valores determinados com XRF como função da fração relativa do $\left[\operatorname{Ir}(\mathrm{ppy})_{3}\right]$ (f) obtida a partir da equação 3.2.

Figura 5.13: Relação entre os valores de concentração em massa determinados pelas técnicas de $R B S\left(f_{R B S}\right)$ e $X R F\left(f_{X R F}\right)$ para filmes codepositados de $\left[\operatorname{lr}(\mathrm{ppy})_{3}\right]:$ CuPc.

Figura 5.14: Espectros de absorção e de emissão a temperatura ambiente dos filmes de [Ir(t-pzp) ${ }_{2}$ pic] (linhas tracejadas) e (a) Spiro2-CBP, (b) TcTa e (c) $\alpha$-NPD (linhas contínuas). ${ }^{1,3} \mathrm{LC}$ e ${ }^{1,3} \mathrm{MLCT}$ referem-se ao acrônimo do Ligante Central e da Transferência de Carga do Metal ao Ligante dos estados singleto e tripleto, respectivamente.

Figura 5.15: Espectros de absorção e emissão a temperatura ambiente dos filmes de Spiro2-CBP (linha tracejada) e (a) [Ir(ppy) $)_{3}$, (b) [Ir(fliq) $\left.2 \mathrm{acac}\right]$, e (c) $\left[\operatorname{lr}(\mathrm{btp})_{2} \mathrm{acac}\right]$ (linha continua).

Figura 5.16: Espectros de emissão dos filmes co-depositados de

(a) Spiro2-CBP, (b) TcTa e (c) $\alpha$-NPD com concentrações ( $x$ ) de 4-12\% em massa do [Ir(t-pzp $)_{2}$ pic]. A linha tracejada indica o pico de emissão do [Ir(t-pzp) ${ }_{2}$ pic] puro. Os valores em vermelho $(y)$ indicam a concentração efetiva determinada através da técnica implementada e discutida na Seção 3.3.3.

Figura 5.17: Esquema de níveis de energia do estado de singleto $\left(S_{1}\right)$ e tripleto $\left(T_{1}\right)$ excitado e do estado de fundamental do singleto $\left(S_{0}\right)$, dos diversos processos de transferência de energia e do processo de emissão de luz no sistema [Ir(t-pzp) $\left.)_{2} p i c\right]: X$, onde X é a) $\alpha$-NPD,

b)Spiro2-CBP, c) TcTa.

Figura 5.18: Espectros normalizados de fotoluminescência $(P L)$ dos filmes de $x \%\left[E u(D B M)_{3} P H E N\right]: S p i r o 2-C B P$, onde $x \%$ é a concentração em massa do dopante na matriz. As diversas curvas referem-se a diferentes concentrações do complexo de Eu na matriz (em vermelho os valores obtidos com a calibração da técnica de co-deposição). No detalhe um aumento dos espectros na região 350-575nm para evidenciar a contribuição das bandas da matriz e do DBM. 
Figura 5.19: Espectros normalizados de fotoluminescência (PL) dos filmes de $x \%\left[E u(D B M)_{3} P H E N\right]: T c T a$, onde $x \%$ é a concentração em massa do dopante na matriz. As diversas curvas referem-se a diferentes concentrações do complexo de Eu na matriz (em vermelho os valores obtidos com a calibração da técnica de co-deposição). No detalhe um aumento dos espectros na região 350-575nm para evidenciar a contribuição das bandas da matriz e do DBM.

Figura 5.20: Espectros normalizados de fotoluminescência $(P L)$ dos filmes de $x \%\left[\mathrm{Eu}(\mathrm{DBM})_{3}(\mathrm{DMSO})_{2}\right]$ :Spiro2-CBP, onde $\mathrm{x} \%$ é a concentração em massa do dopante na matriz. As diversas curvas referem-se a diferentes concentrações do complexo de Eu na matriz (em vermelho os valores obtidos com a calibração da técnica de co-deposição). No detalhe um aumento dos espectros na região 350-575nm para evidenciar a contribuição das bandas da matriz e do $(\mathrm{DBM})_{3}(\mathrm{DMSO})_{2}$.

Figura 5.21: Espectros normalizados de fotoluminescência $(P L)$ dos filmes de $x \%\left[E u(D B M)_{3}(D M S O)_{2}\right]: T c T a$, onde $x \%$ é a concentração em massa do dopante na matriz. As diversas curvas referem-se a diferentes concentrações do complexo de Eu na matriz (em vermelho os valores obtidos com a calibração da técnica de co-deposição). No detalhe um aumento dos espectros na região 350-575nm para evidenciar a contribuição das bandas da matriz e do $(\mathrm{DBM})_{3}(\mathrm{DMSO})_{2}$.

Figura 5.22: Diagrama parcial de energia e os processos de transferência de energia por excitação óptica, dos filmes co-depositados de (a) $\left[\mathrm{Eu}(\mathrm{DBM})_{3} \mathrm{PHEN}\right]:$ Spiro2-CBP, $\left[\mathrm{Eu}(\mathrm{DBM})_{3}(\mathrm{DMSO})_{2}\right]: \mathrm{TcTa}$ e (b) $\left[\mathrm{Eu}(\mathrm{DBM})_{3} \mathrm{PHEN}\right]: \mathrm{TcTa},\left[\mathrm{Eu}(\mathrm{DBM})_{3}(\mathrm{DMSO})_{2}\right]:$ Spiro2-CBP.

Figura 5.23: Estrutura do PHOLED de [Ir(t-pzp) ${ }_{2}$ pic] no diagrama rígido de bandas de energia das diferentes camadas orgânicas. Para os eletrodos (ITO e Al) é indicada a energia da função de trabalho e para os orgânicos os níveis de HOMO e LUMO.

Figura 5.24: (a) Comparação entre os espectros de PL do filme de $\left[\operatorname{Ir}(\mathrm{t}-\mathrm{pzp})_{2} \mathrm{pic}\right]$ e de EL do PHOLED; (b) diagrama de cromaticidade CIE para as emissões de PL e EL.

Figura 5.25: Curvas de luminância e de eficiência do PHOLED [Ir(t-pzp $)_{2}$ pic]. No detalhe uma foto do PHOLED a $18 \mathrm{~V}$. 
Figura 5.26: (a) Estrutura dos PHOLEDs dos $\mathrm{x} \%$ em massa de [ $\left.\operatorname{lr}(\mathrm{t}-\mathrm{pzp})_{2} \mathrm{pic}\right]: Y$ sendo Y: Spiro2-CBP ou TcTa e os valores "a" e "b" variam dependendo do HOMO e LUMO do Spiro2-CBP ou TcTa (Tabela 5.6), no diagrama rígido de bandas de energia das diferentes camadas orgânicas. Para os eletrodos (ITO e Al) é indicada a energia da função trabalho e para os orgânicos os níveis de HOMO e LUMO, (b) HOMO e LUMO do complexo e das matrizes.

Figura 5.27: Espectros de EL e diagramas de cromaticidade CIE dos dispositivos PHOLEDs de [Ir(t-pzp) ${ }_{2}$ pic] dopado em (a) Spiro2-CBP, (b) TcTa e (c) $\alpha$-NPD com diferentes concentrações em massa.

Figura 5.28: Espectros de eletroluminescência em função da tensão aplicada para os PHOLEDs de (a) [Ir(t-pzp) ${ }_{2}$ pic]:Spiro2-CBP e (b) $\left[\operatorname{lr}(\mathrm{t}-\mathrm{pzp})_{2} \mathrm{pic}\right]:$ TcTa.

Figura 5.29: Características L-V dos PHOLEDs baseados em $\% x\left[\operatorname{lr}(\mathrm{t}-\mathrm{pzp})_{2} \mathrm{pic}\right]: Y$, onde $\mathrm{Y}$ é (a) Spiro2-CBP e (b) TcTa. Os valores em vermelho são os valores efetivos da concentração do dopante calculados usando a calibração da seção 5.2.2.3. Abaixo de cada curva L-V é mostrada a foto do melhor dispositivo a baixa tensão e sua respectiva luminância.

Figura 5.30: Curvas de eficiência em (Im/watt) e de eficiência quântica externa (EQE) dos melhores PHOLEDs baseados em [Ir(t-pzp) $)_{2}$ pic] em Spiro2-CBP e TcTa. Abaixo do gráfico são mostrados as fotos dos dois tipos de PHOLEDs para uma mesma densidade de corrente de $10 \mathrm{~mA} / \mathrm{cm}^{2}$.

Figura 5.31: Espectros de EL dos PHOLEDs (a) 8\%[Ir(t-bupzp) 3 :

Spiro2-CBP e (b) 14\% [Ir(t-bupzp) $\left.)_{3}\right]$ :Spiro2-CBP em função da tensão aplicada. Em (c) é mostrado o diagrama de cromaticidade CIE para os espectros de EL a $20 \mathrm{~V}$ de (a) e (b).

Figura 5.32: Comparação entre os espectros de PL do [fac-Ir(t-bupzp) ${ }_{3}$ ] em solução de diclorometano $\left(10^{-5} \mathrm{~mol} / \mathrm{L}\right)^{[186]}$ e da EL do dispositivo 4 . Os espectros foram registrados a temperatura ambiente. Na direita a foto do dispositivo a $18 \mathrm{~V}$.

Figura 5.33: Diagrama de energia e processos de transferência de

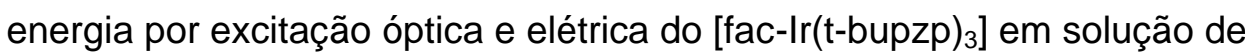
diclorometano $\left(10^{-5} \mathrm{~mol} / \mathrm{L}\right)^{[186]}$ e da EL do dispositivo 4, respectivamente. 
Figura 5.34: Curvas de luminância $\left(\mathrm{cd} / \mathrm{m}^{2}\right)$ e eficiência $(\mathrm{Im} / \mathrm{W})$ em função da voltagem e da densidade da corrente (J) dos PHOLEDs de [fac-Ir(t-bupzp) $\left.)_{3}\right]:$ Spiro2-CBP.

Figura 5.35: Espectros de abosorção e emissão dos filmes dos complexos de (a) [Ir(fliq) ${ }_{2}$ acac] e (b) [Ir(btp) ${ }_{2}$ acac].

Figura 5.36: Espectro de EL de: (a) dispositivo 6 baseado em [Ir(btp) $)_{2}$ acac] $\left(x_{0}\right)$ e dispositivo 7 com $10 \%$ do mesmo complexo em Spiro2-CBP $\left(\mathrm{x}_{1}\right)$ e TcTa $\left(\mathrm{x}_{2}\right)$; (b) dispositivo 6 baseado em [Ir(fliq) $)_{2}$ acac] $\left(\mathrm{x}_{0}\right)$ e dispositivo 7 com $7 \%$ do mesmo complexo em Spiro2-CBP $\left(x_{1}\right)$ e TcTa $\left(x_{2}\right)$.

Figura 5.37: Estrutura do dispositivo 6 para os PHOLEDs de [Ir(btp) $)_{2}$ acac] ou [Ir(fliq) $)_{2}$ acac] no diagrama rígido de bandas de energia das diferentes camadas orgânicas. Para os eletrodos (ITO e Al) é indicada a energia da função de trabalho e para a parte orgânica os níveis de HOMO e LUMO.

Figura 5.38: Diagrama de bandas de energia e os processos de transferência de energia dos $\mathrm{PHOLEDs}$ de $\operatorname{Ir}(\mathrm{btp})_{2}$ acac:Spiro2-CBP e $\operatorname{Ir}(\text { btp })_{2}$ acac:TcTa.

Figura 5.39: (a) Luminância $\left(\mathrm{cd} / \mathrm{m}^{2}\right)$, (b) eficiência (Im/W) e EQE \% dos PHOLEDs de $10 \%\left[\operatorname{lr}(\text { btp })_{2}\right.$ acac]:Y e $7 \%\left[\operatorname{lr}(\text { fliq })_{2}\right.$ acac]:Y, onde $Y$ são as matrizes Spiro2-CBP e TcTa. A direita as fotos dos PHOLEDs baseados na matriz Spiro2-CBP para uma mesma densidade de corrente de $1,4 \mathrm{~mA} / \mathrm{cm}^{2}$.

Figura 5.40: Curvas de (a) luminância e (b) eficiência para PHOLEDs com diferentes concentrações de $\left[\mathrm{Eu}(\mathrm{DBM})_{3} \mathrm{PHEN}\right]$ em Spiro2-CBP. Em (c) é mostrado o diagrama de cromaticidade CIE e as fotos dos PHOLEDs para as três concentrações de $\left[\mathrm{Eu}(\mathrm{DBM})_{3} \mathrm{PHEN}\right]$ para uma densidade corrente de $1,2 \mathrm{~mA} / \mathrm{cm}^{2}$.

Figura 5.41: Estrutura do dispositivo 8 para o PHOLED de $\left[\mathrm{Eu}(\mathrm{DBM})_{3} \mathrm{PHEN}\right]:$ Spiro2-CBP no diagrama rígido de bandas de energia das diferentes camadas orgânicas. Para os eletrodos (ITO e Al) é indicada a energia da função de trabalho e para os orgânicos os níveis de HOMO e LUMO. 
Figura 5.42: Espectro de EL do PHOLED com $11,2 \%\left[\mathrm{Eu}(\mathrm{dbm})_{3}\right.$ phen] em Spiro2-CBP em função da tensão aplicada. No detalhe o aumento da região entre 350 e 600nm onde é possível notar a contribuição da emissão das outras camadas. Ao lado direito o espectro da PL a 77K do complexo $\left[\mathrm{Gd}(\mathrm{DBM})_{3}(\mathrm{DMSO})_{2}\right]^{[57]}$ que mostra a banda de fosforescência do ligante DBM.

Figura 5.43: Curvas de (a) luminância e (b) eficiência para PHOLEDs com diferentes concentrações de $\left[\mathrm{Eu}(\mathrm{DBM})_{3} \mathrm{PHEN}\right]: \mathrm{TcTa}$. Em (c) é mostrado o diagrama de cromaticidade CIE e as fotos dos PHOLEDs $\left[\mathrm{Eu}(\mathrm{DBM})_{3} \mathrm{PHEN}\right]$ para uma densidade corrente de $1 \mathrm{~mA} / \mathrm{cm}^{2}$.

Figura 5.44: Espectro de EL do PHOLED com 15,3\%[Eu(DBM) $\left.{ }_{3} \mathrm{PHEN}\right]$ em TcTa em função da tensão aplicada. No detalhe o aumento da região entre 350 e 600nm onde é possível notar a contribuição da emissão das outras camadas.

Figura 5.45: Espectros normalizados de EL dos PHOLEDs baseados em [Eu(DBM $)_{3}$ phen], [Eu(DBM $)_{3}(\mathrm{DMSO})_{2}$ ] e dos dispositivos baseados em $15,3 \%$ em massa de $\left[\mathrm{Eu}(\mathrm{DBM})_{3}\right.$ phen]:Y e $\left[\mathrm{Eu}(\mathrm{DBM})_{3}(\mathrm{DMSO})_{2}\right]: Y$, sendo $\mathrm{Y}$ as matrizes de Spiro2-CBP e TcTa. No lado as fotos dos PHOLEDs de $\left.15,3 \%\left[\mathrm{Eu}(\mathrm{DBM})_{3} \text { phen]:Y e 15,3\%[Eu(DBM }\right)_{3}(\mathrm{DMSO})_{2}\right]: Y$, sendo $\mathrm{Y}$ as matrizes de Spiro2-CBP e TcTa com uma densidade de corrente de $1 \mathrm{~mA} / \mathrm{cm}^{2}$.

Figura 5.46: Curvas de (a) luminância e (b) eficiência dos PHOLEDs baseados em $\left[\mathrm{Eu}(\mathrm{DBM})_{3} \mathrm{PHEN}\right]$, $\left[\mathrm{Eu}(\mathrm{DBM})_{3}(\mathrm{DMSO})_{2}\right]$ e $15,3 \%$ $\left[\mathrm{Eu}(\mathrm{DBM})_{3} \mathrm{PHEN}\right]: \mathrm{Y}$ e $15,3 \%\left[\mathrm{Eu}(\mathrm{DBM})_{3}(\mathrm{DMSO})_{2}\right]: \mathrm{Y}$, sendo $\mathrm{Y}$ as matrizes de Spiro2-CBP e TcTa.

Figura 5.47: Imagens $\operatorname{AFM}(3 \times 3 \mu \mathrm{m})$ dos filmes de $70 \mathrm{~nm}$ de $\mathrm{Alq}_{3}$ depositados termicamente sobre (a) substrato de silício que não foi submetido a vibração; e sobre substratos de silício submetidos a vibração de: (b) $50 \mathrm{~Hz}$, (c) $100 \mathrm{~Hz}$, (d) $300 \mathrm{~Hz}$, (e) $800 \mathrm{~Hz}$. (f) e (g) são imagens $A F M(1 \mu \mathrm{m} \times 1 \mu \mathrm{m})$ em 3D da área indicada pela seta em (a) e da imagem $(\mathrm{d})$, respectivamente.

Figura 5.48: Imagens $A F M$ de filmes orgânicos depositados termicamente com uma espessura de 70nm de (a) Alq $_{3}$ e (b) $\beta$-NPB, sobre substratos de silício sem vibrar e substrato de silício vibrando $(100 \mathrm{~Hz})$. 
Figura 5.49: Observação e análise do filme com 10nm de espessura e 10\% em massa do $\left[\operatorname{Ir}(\mathrm{ppy})_{3}\right]$ em Spiro2-CBP: (a) Imagem por campo claro no modo TEM do filme; nas regiões 1,2 e 3 observa-se diferenças no contraste do filme de $\left[\operatorname{lr}(\mathrm{ppy})_{3}\right]$ :Spiro2-CBP, sendo a região 3 a zona que não tem filme, (b) Espectro EDS na região indicada no quadrado 1 confirmando que a amostra possui Irídio. A presença de Cu e parte do $\mathrm{C}$ é devido à grade utilizada como substrato.

Figura 5.50: Micrografia e análise EDS do filme de 40nm de espessura e concentração de $10 \%$ em massa de $\left[\operatorname{Ir}(\mathrm{ppy})_{3}\right]$ em Spiro2-CBP. (a) Imagem por campo escuro (HAADF) no modo STEM do filme. (b) Espectros EDS nas regiões indicadas nos quadrados 1,3 e 4 confirmando que a amostra possui irídio. A presença de Cu é devida à grade usada como substrato.

Figura 5.51: Observação e análise EDS do filme de 40nm de espessura e concentração de $30 \%$ em massa de $\left[\operatorname{lr}(\mathrm{ppy})_{3}\right]$ em Spiro2-CBP. (a) e (b) Imagens por campo escuro (HAADF) no modo STEM. Abaixo de cada imagem são reportados os respectivos espectros $E D S$ das regiões indicadas nos quadrados confirmando a presença de Irídio. A presença de Cu é devida à grade usada como substrato.

Figura 5.52: Observação e análise EDS do filme de 40nm de espessura e concentração de 30\% em massa do [Ir(ppy) $)_{3}$ ] em Spiro2-CBP. (a) e

(b) Imagens por campo escuro (HAADF) no modo STEM do filme e

(b) Espectros $E D S$ das regiões 1 e 2 da micrografia (b), confirmando que a amostra possui Irídio. A presença de Cu é devida à grade do substrato.

Figura 5.53: Análises de varreduras lineares no modo STEM do filme de 40nm de espessura e concentração de $30 \%$ em massa do $\left[\operatorname{Ir}(\mathrm{ppy})_{3}\right]$ em Spiro2-CBP. (a) Imagem ( $\left.7 \times 7 \mu \mathrm{m}^{2}\right)$ por campo escuro (HAADF) no modo STEM, (b) Curvas do sinal de $\operatorname{Ir}($ linha $M \alpha$ ) espalhado e medido pelo detector HAADF em função da posição do filme, e (c) Curvas do sinal $E D S$ em função da posição do filme.

Figura 5.54: Análises de varreduras lineares no modo STEM do filme de 40nm de espessura e concentração de $30 \%$ em massa do $\left[\operatorname{lr}(\text { ppy })_{3}\right]$ em Spiro2-CBP usando a técnica de vibração do substrato $(100 \mathrm{~Hz})$. (a) Imagem $\left(7 x 7 \mu \mathrm{m}^{2}\right)$ por campo escuro (HAADF) no modo STEM, (b) Curvas do sinal de Ir (linha $M \alpha$ ) espalhados e medidos pelo detector $H A A D F$ em função da posição do filme. 
Figura 5.55: Estrutura do OLED com emissão no verde usado em este estudo, indicando os níveis de energia dos materiais orgânicos.

Figura 5.56: Curvas luminância - tensão dos OLEDs fabricados em dois condições experimentais diferentes: (a) só o filme de $\mathrm{Alq}_{3}$ foi depositado com o substrato vibrando, (b) os filmes de $\mathrm{Alq}_{3}$ e $\beta$-NPB foram depositados com o substrato vibrando.

Figura 5.57: Curvas da eficiência dos OLEDs para duas condições diferentes: (a) somente o filme de $\mathrm{Alq}_{3}$ foi depositado com o substrato vibrando (100 Hz), (b) os filmes de $\mathrm{Alq}_{3}$ e $\beta$-NPB foram depositados com o substrato vibrando $(100 \mathrm{~Hz})$.

Figura 5.58: Espectros de eletroluminescência dos PHOLEDs de 7\%[Ir(fliq) $)_{2}$ acac] em Spiro2-CBP e TcTa, com e sem LiF.

Figura 5.59: (a) Estrutura do dispositivo 10 no diagrama rígido de bandas de energia das diferentes camadas orgânicas. Para os eletrodos (ITO e Al) é indicada a energia da função de trabalho e para os orgânicos os níveis de HOMO e LUMO e (b) Diagrama de bandas de energia e processos de transferência de energia dos $P H O L E D$ s de $\operatorname{Ir}(\text { fliq })_{2}$ acac:Spiro2-CBP e $\operatorname{Ir}(\text { fliq })_{2}$ acac:TcTa.

Figura 5.60: Curvas de luminância $\left(\mathrm{cd} / \mathrm{m}^{2}\right)$ e eficiência em função da tensão e densidade de corrente, respectivamente, dos PHOLED de 7\%[Ir(fliq) ${ }_{2}$ acac] em Spiro2-CBP e TcTa, com e sem LiF.

Figura 5.61: (a) Espectros de EL do dispositivo 11 em função da tensão aplicada, (b) coordenadas de CIE do dispositivo em função da tensão aplicada e (c) fotos do PHOLED com diferentes tensões aplicadas ao dispositivo.

Figura 5.62: (a) Espectros de EL do dispositivo 11 em função da tensão aplicada, (b) coordenadas de CIE do dispositivo em função da tensão aplicada e (c) fotos do PHOLED com diferentes tensões aplicadas ao dispositivo.

Figura 5.63: (a) Espectros de EL do dispositivo 13 em função da tensão aplicada, (b) coordenadas de CIE do dispositivo em função da tensão aplicada e (c) fotos do PHOLED com diferentes tensões aplicadas ao dispositivo. 


\section{Lista de tabelas}

Tabela 3.1: Complexos usados na camada emissora como dopantes fosforescentes

62

Tabela 3.2: Compostos orgânicos usados na camada emissora como matrizes orgânicas

Tabela 3.3: Compostos orgânicos denominados como transportadores de carga nos dispositivos OLEDs.

Tabela 3.4: Compostos orgânicos usados como injetores ou bloqueadores de carga na fabricação dos OLEDs.

Tabela 4.1: Tabela de conversão fotopica para diferentes comprimentos de onda [152,153].

Tabela 5.1: Emissão do estado de tripleto dos ligantes: ppz, t-pzp, e ppy

Tabela 5.2: Tempo de vida dos complexos a) [Ir(tpzp)2pic], b) [Ir(ppy)3],

c) [Ir(btp)2acac] e d) [Ir(fliq)2acac].

Tabela 5.3: Modos de vibração e freqüência do FTIR de [Ir(t-pzp)2pic]

e $[\operatorname{lr}(\mathrm{ppz}) 3]$

Tabela 5.4: Dados dos níveis de energia dos seguintes complexos fosforescentes: [Ir(t-pzp)2pic], [Ir(ppy)3], [Ir(fliq)2acac], e [Ir(btp)2acac].

Tabela 5.5: Gap óptico (Eg), estado de singleto (Es) e estado do tripleto (ET) das matrizes utilizadas na fabricação dos PHOLEDs.

Tabela 5.6: Valores de HOMO e LUMO para as diversas matrizes estudadas.

Tabela 5.7: Resultados obtidos na análise dos espectros RBS para a determinação do numero de átomos de Cu e Ir nos filmes finos co-depositados com diferentes concentração de [Ir(ppy)3] em CuPc. O $f$ é a fração relativa do [Ir(ppy)3] determinada com a técnica de co-deposição térmica e $\varepsilon$ é o erro entre os dados experimentais ( $\mathrm{Ir} / \mathrm{Cu}$ ) obtidos com RBS em (at/cm2) e [g] e o $f$ os dados da técnica de co-deposição.

Tabela 5.8: Valores da concentração relativa de $[\operatorname{Ir}(\mathrm{ppy}) 3](f)$ comparadas com os valores da concentração em massa de [Ir(ppy)3] obtidas com as técnicas de XRF e RBS para filmes co-depositados de $\operatorname{Ir}(p p y) 3: C u P c$. 
Tabela 5.9: Valores da concentração relativa do $[\operatorname{Ir}(\mathrm{ppy}) 3](f)$ comparadas com os valores da concentração em massa do [Ir(ppy)3] para filmes codepositados de [Ir(ppy)3]:Spiro2-CBP obtidos com as técnicas XRF e o RBS calculados a partir do gráfico da Figura 5.13.

Tabela 5.10: Raio de Förster (R0) para a análises da transferência de energia entre Spiro2-CBP, TcTa e $\alpha$-NPD e [Ir(t-pzp)2pic]. O valor de R0 do CBP foi obtido da literatura [200].

Tabela 5.11: Resumo dos resultados para os PHOLEDs baseados no [ $\operatorname{lr}(\mathrm{t}-\mathrm{pzp}) 2 \mathrm{pic}]$. Os valores foram medidos para uma densidade de corrente de $10 \mathrm{~mA} / \mathrm{cm} 2$.

Tabela 5.12: Valores da rugosidade quadrática media (RMS) dos filmes de Alq3 em função da frequências de vibração do substrato de silício.

Tabela 5.13: Valores da rugosidade RMS dos filmes depositados termicamente de Alq3 e $\beta$-NPB com uma taxa de deposição de $0,1 \mathrm{~nm} / \mathrm{s}$ com o substratos de silício sem vibrar e substratos de silício com vibração fixa $(100 \mathrm{~Hz})$.

Tabela 5.14: Valores da rugosidade RMS dos filmes de Alq3 e $\beta$-NPB com diferentes taxas de deposição com o substratos de silício vibrando a $100 \mathrm{~Hz}$.

Tabela 5.15: Valores meios dos sinais receptadas pelos detectores HAADF e EDS

Tabela 5.16: Valores meios dos sinais receptados pelos detectores EDS e HAADF usando a técnica de vibração do substrato na co-deposição do filme $[\operatorname{lr}($ ppy)3]:Spiro2-CBP. 


\section{Lista de Símbolos e Abreviações}

AFM - microscopia de força atômica

EDS - espectroscopia por energia dispersiva

EQI - eficiência quântica interna

EL - eletroluminescência

$f$ - fração de concentração do dopante/composto emissor na matriz/composto emissor num filme fino co-depositado termicamente.

$f_{\mathrm{RBS}}$ - concentração em peso do dopante determinada pela técnica RBS

$f_{\mathrm{XRF}}$ - concentração em peso do dopante determinada pela técnica XFR

FTIR - espectroscopia no infravermelho

HADAF - detector de campo escuro anular de grande ângulo

HOMO - orbital molecular ocupado mais alto

ISC - cruzamento inter-sistemas

$\mathrm{k}_{\mathrm{d}}$ - taxa de decaimento radiativo do doador

$\mathrm{k}_{\mathrm{a}}$ - taxa de decaimento radiativo do receptor

LC - ligante central

LUMO - orbital molecular de mais baixa energia

MLCT - transferência de carga metal-ligante.

OLED - Dispositivos emissor de luz orgânico, do inglês Organic Light-emitting diode

PHOLEDs - Oleds baseados em complexos fosforescentes, do inglês Phosphorescent organic light emitting diode

QCM - microbalança de cristal de quartzo

RBS - espectroscopia de retro-espalhamento Rutherford

SOC - acoplamento spin-órbita

STEM - microscópio eletrônico de varredura por transmissão

$\mathrm{T}_{\mathrm{g}}$ - taxa de deposição térmica do dopante

$\mathrm{T}_{\mathrm{h}}$ - taxa de deposição térmica da matriz

TEM - microscopia eletrônica de transmissão

WOLEDs - Oleds com emissão de luz branca, do inglês White organic light emitting diode.

XFR - fluorescência de raios-X 